\title{
A graphic way for notch shape optimization
}

\author{
C. Mattheck, J. Sörensen \& K. Bethge \\ Institute for Materials Research II, \\ Forschungszentrum Karlsruhe GmbH, Germany
}

\begin{abstract}
Many components have notches and notches are in the majority of cases the reason for failure. There are many options to reach a longer lifetime and a better utilisation of material. One method for the shape optimization of components, developed in Forschungszentrum Karlsruhe, uses the design rules of nature. During the last 15 years it has spread very well and proven itself in industry, especially in automotive engineering. The limits for using the CAO-method (Computer Aided Optimization) are more or less of an economic nature. So we need a method that allows optimization by everybody.

Now a new pure graphical method has been found, which works without any FEM or optimization software. It is called the "Method of Tensile Triangles" and requires only a set square and a piece of paper to optimize the notch shape in an effective and simple way.
\end{abstract}

Keywords: optimization of components, design rules, fatigue, graphical methods.

\section{Introduction}

It is of constantly increasing significance for industry to save time and money in order to produce more efficiently. The time to construct or modify components becomes shorter and shorter at the end of a development phase. Consequently, it is not always possible to apply complex, FE-based optimization methods. Such methods are mainly and justifiably used in special problem cases or highly loaded areas. The CAO method (computer-aided optimization) [1] eases problematic points, but often requires several iterations. In case of complex components, use of the CAO method together with FEM analyses is associated with the need for computing capacity and time. 
In many cases, the "method of tensile triangles" would rapidly produce good results, and this only with a set square or with simple construction rules in CAD.

\section{Procedure of the "Method of Tensile Triangles"}

When studying the shoulder fillet under tensile loading (Fig. 1), potential slip planes and shear stresses due to an obvious longitudinal shear result in equivalent tensile stresses. This results in principle tensile stresses that are tilted by about $45^{\circ}$ to the beam axis near the notch.

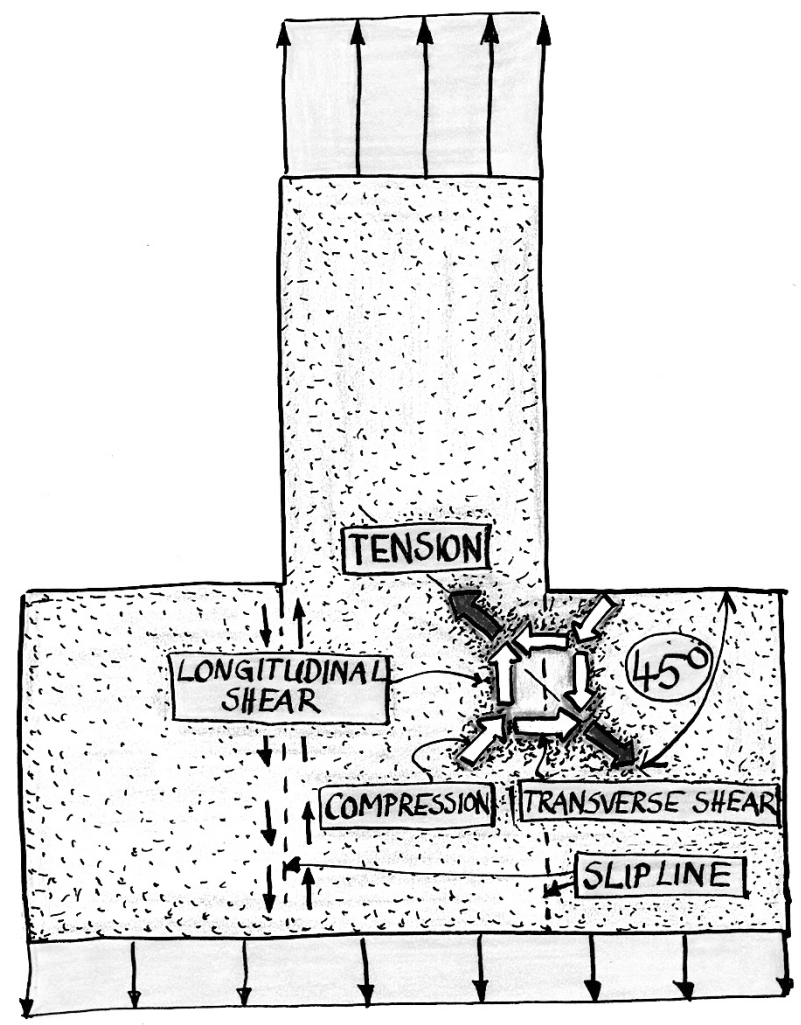

Figure 1: $\quad$ Stresses near a notch under tensile loading.

The "method of tensile triangles" starts with this knowledge. When looking at the foot of a tree's trunk, see Fig. 2, a $45^{\circ}$ tension rope is found to extend along the upper side of the root. When applied to technical components, an existing notch is bridged by a tensile triangle, as shown in Fig. 3. The repetition of this procedure is the method of tensile triangles. Figure 3 illustrates the method proposed by Mattheck and verified for a shoulder fillet in [2]. In a last step, the segments have to be rounded to obtain an optimized notch shape. 

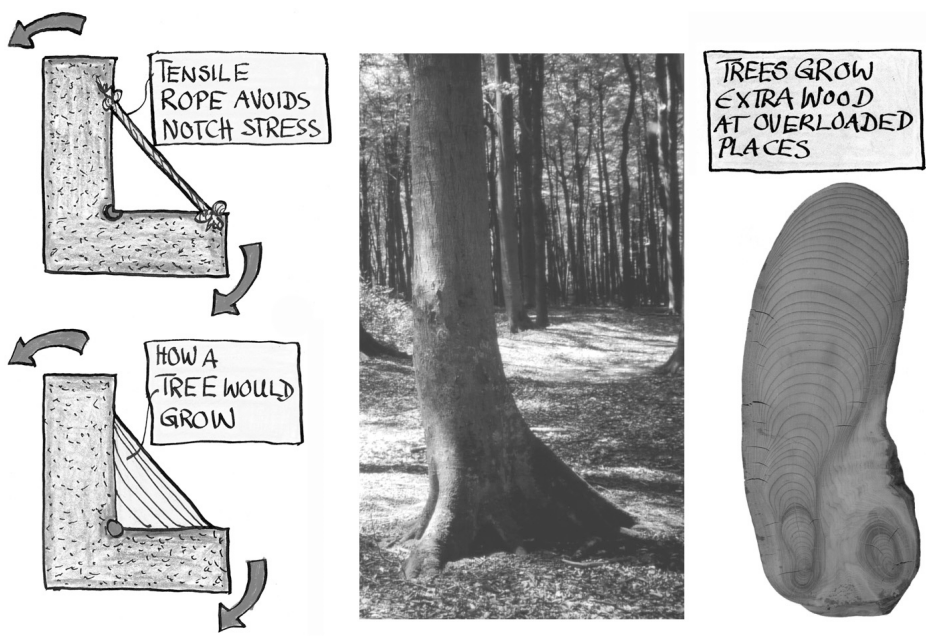

Figure 2: The buttress root bridges the sharp edge at the foot of the trunk like a rope.

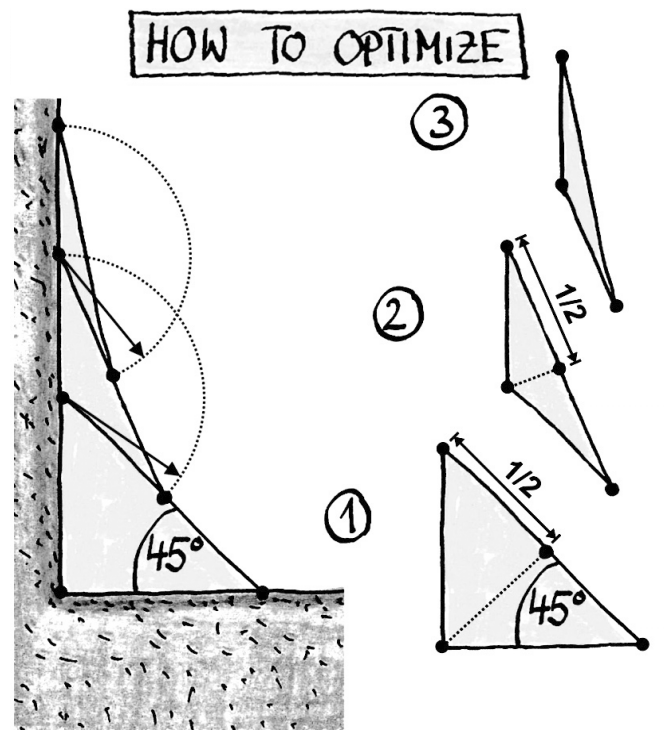

Figure 3: $\quad$ Principle of the method of tensile triangles for uniaxial tension.

\section{Examples of application}

\subsection{Shoulder fillet}

The optimization method is aimed at reducing notch stresses of components. These notch stresses are locally highly loaded areas which often represent the 
starting points of cracks in the component and, hence, the start of failure. By optimizing the geometry of such notches, it is possible to reduce the notch stresses so that component lifetime is increased significantly.

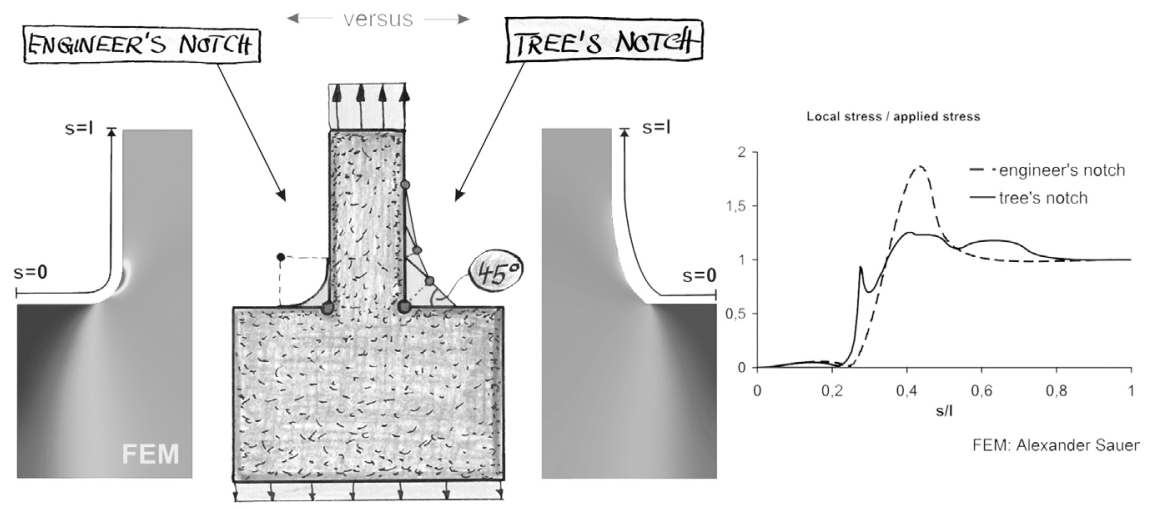

Figure 4: $\quad$ Stresses at the shoulder fillet. Rounding with a quadrant notch (left) and method of tensile tri-angles (right).

It is obvious from Fig. 4 that the shoulder fillet on the left that is rounded with a quadrant notch causes a locally high stress maximum. In contrast to this, the shoulder optimized by means of the method of tensile triangles is characterized by a very homogeneous stress along the contour.

\subsection{Fork}

Biaxial loads of forks e.g. may also be optimized by means of the method of tensile triangles (Figs. 5 and 6) [2]. At the same load ratios, a point of intersection of the bisecting line with the perpendiculars to the legs is obtained. From this point of intersection, the first tensile triangle is generated at an angle of $45^{\circ}$ to the perpendicular. The following tensile triangles bridge the newly generated notches with increasingly obtuse angles and, thus, increasingly ease the notches.

Each leg with the corresponding perpendicular forms a shoulder and is optimized in analogy to the shoulder fillet.

At unbalanced load ratios, the bisecting line from Fig. 5 varies depending on the ratios. At a load ratio of 3 to 1 , for instance, it changes to a ratio of $45^{\circ}$ to $15^{\circ}$, as shown in Fig. 6. Variation of this ratio also changes the design space for the corresponding tensile triangles. The way of designing does not change. As described above, the individual straight lines are smoothed by radii.

As is obvious from the stress plot (Fig. 6), maximum stress of the stress curve is significantly reduced.

A common question in the optimization of a notch shape is "how large shall the design spaces be chosen?". To answer this question, it can be stated that 
irrespective of the other parameters, always the largest possible design space should be chosen in principle. In most cases, this "largest possible" design space is limited by the component function or accessory parts.
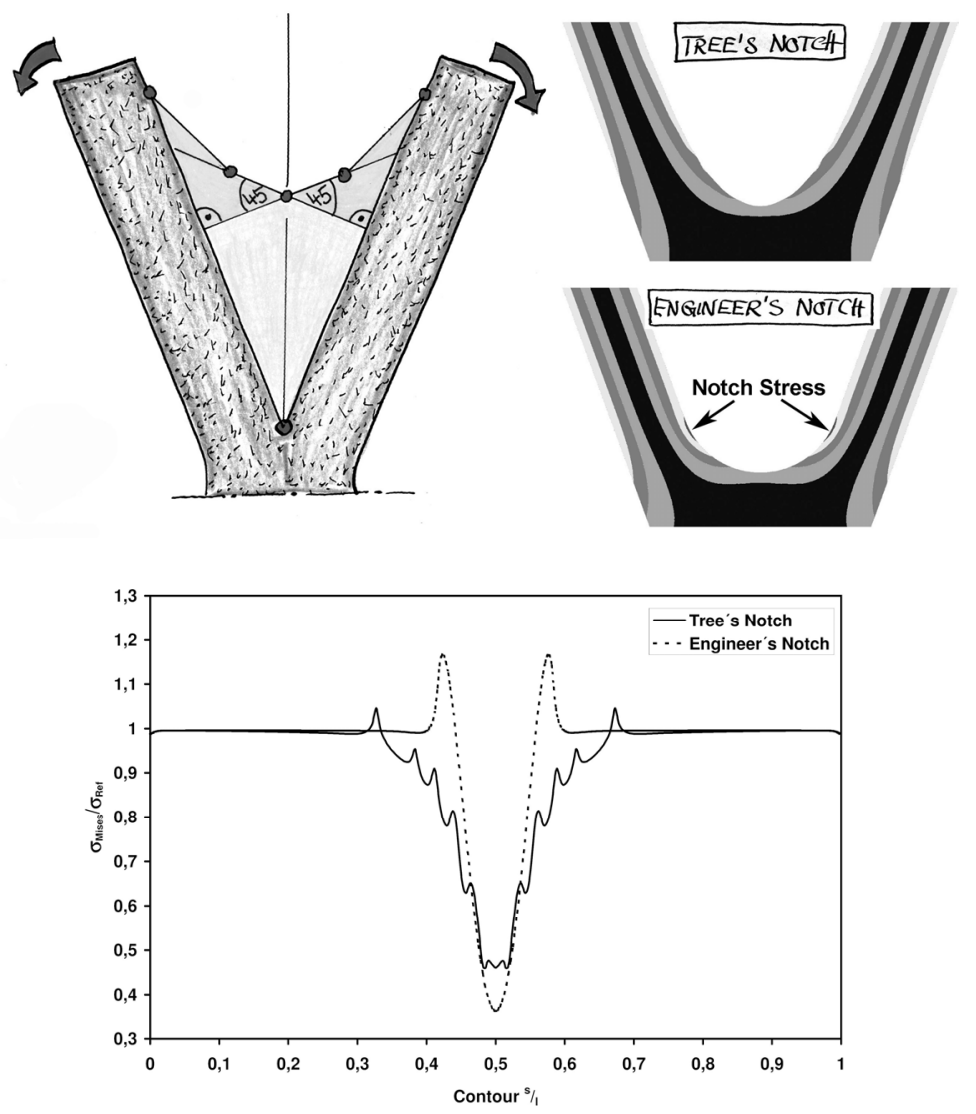

Figure 5: $\quad$ Fork under symmetric bending load.

To determine the design space required for a stress concentration factor of about 1, a FE model of the beam shoulder was studied using various radial design spaces. The geometry for all models studied is a beam shoulder under tensile loading with a width ratio of 3 to 1 . Figure 7 shows the results of this study compared to the optimization by CAO. For the beam shoulder selected here, models with a radial design space of more than $45 \%$ of the largest possible design space, i.e. the width of the shoulder, exhibit a maximum stress similar to CAO optimization shown in Fig. 7. Furthermore, it can be noticed that an increase of the design space by more than $45 \%$ results in a small reduction of the stress only, whereas the same increase of the design space at lower levels causes high stress reductions. 
18 Design and Nature III: Comparing Design in Nature with Science and Engineering
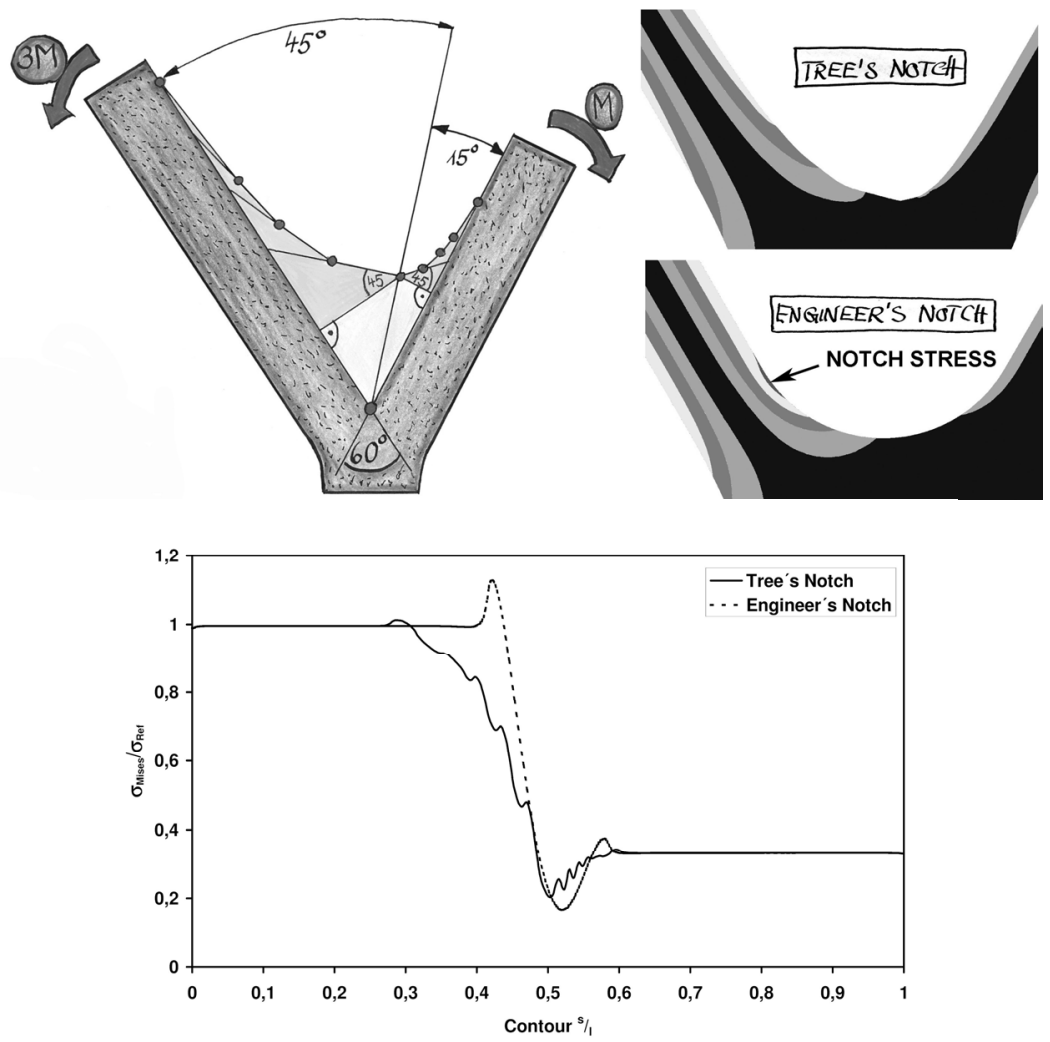

Figure 6: $\quad$ Fork under unsymmetrical bending load (3 to 1).

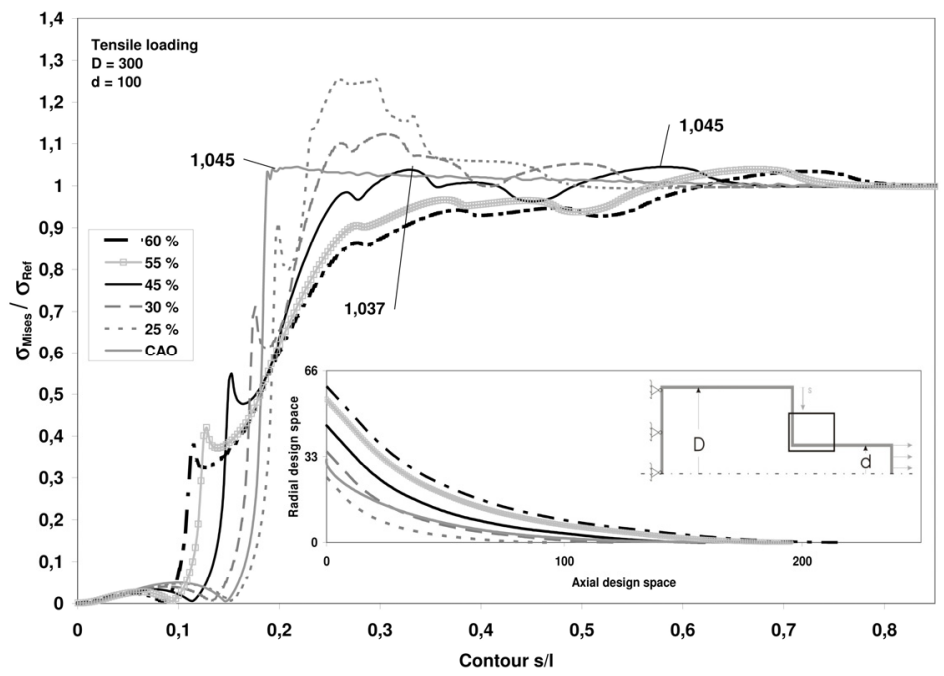

Figure 7: Studies related to the radial design space. 


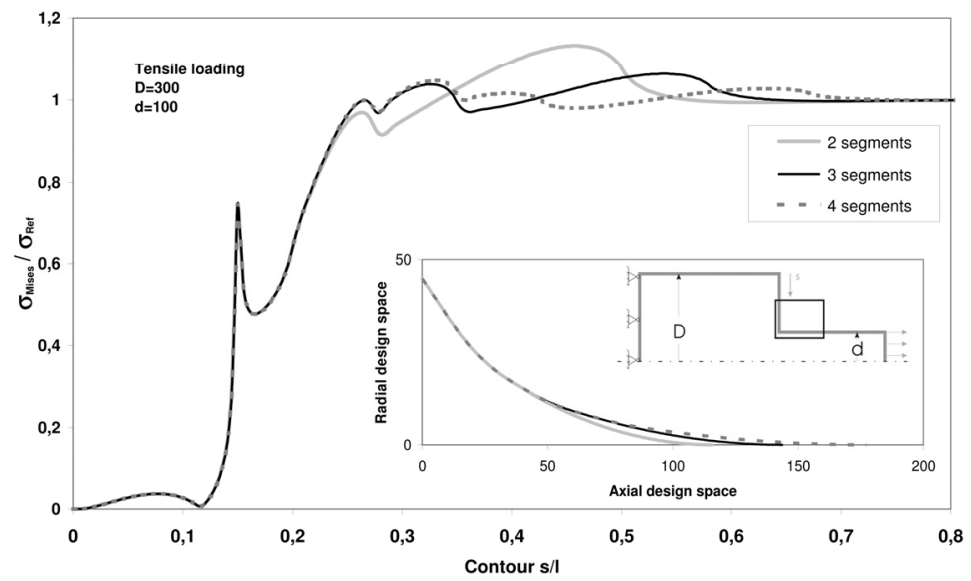

Figure 8: $\quad$ Studies related to the axial design space.

When looking at the number of tensile triangles used to determine the axial design space needed (see Fig. 8), it becomes obvious that the use of more than three tensile triangles does not reduce the stresses any further, but increases the required axial design space. Hence, not less than two, but, if possible, three tensile triangles should be used.

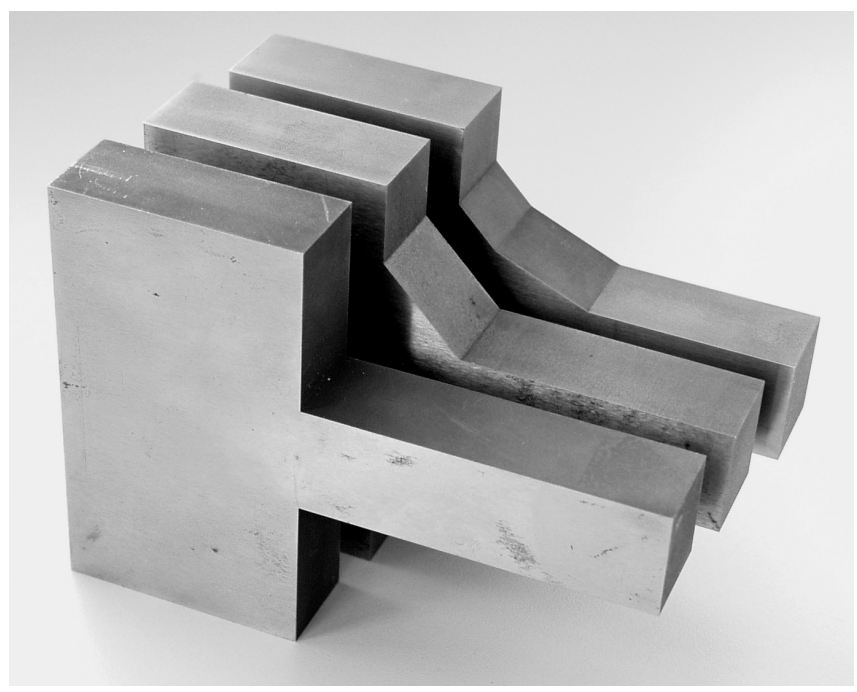

Figure 9: Non-optimized specimen (front) and two specimen with different degree of optimization. 


\section{Experiments}

To verify the method of tensile triangles, beam shoulders were subjected to fatigue tests using a variable number of tensile triangles [3]. Figure 9 shows the specimen types and Fig. 10 the results of the fatigue tests. It is obvious that the notch located on the right side in the figure is always the most endangered one and that the initiation path of the crack is located vertically to the "tension rope" of the following tensile triangle. This makes the method of tensile triangles plausible. At the position of the potential crack, most material is applied and the tensile rope bridges the potential crack symmetrically.
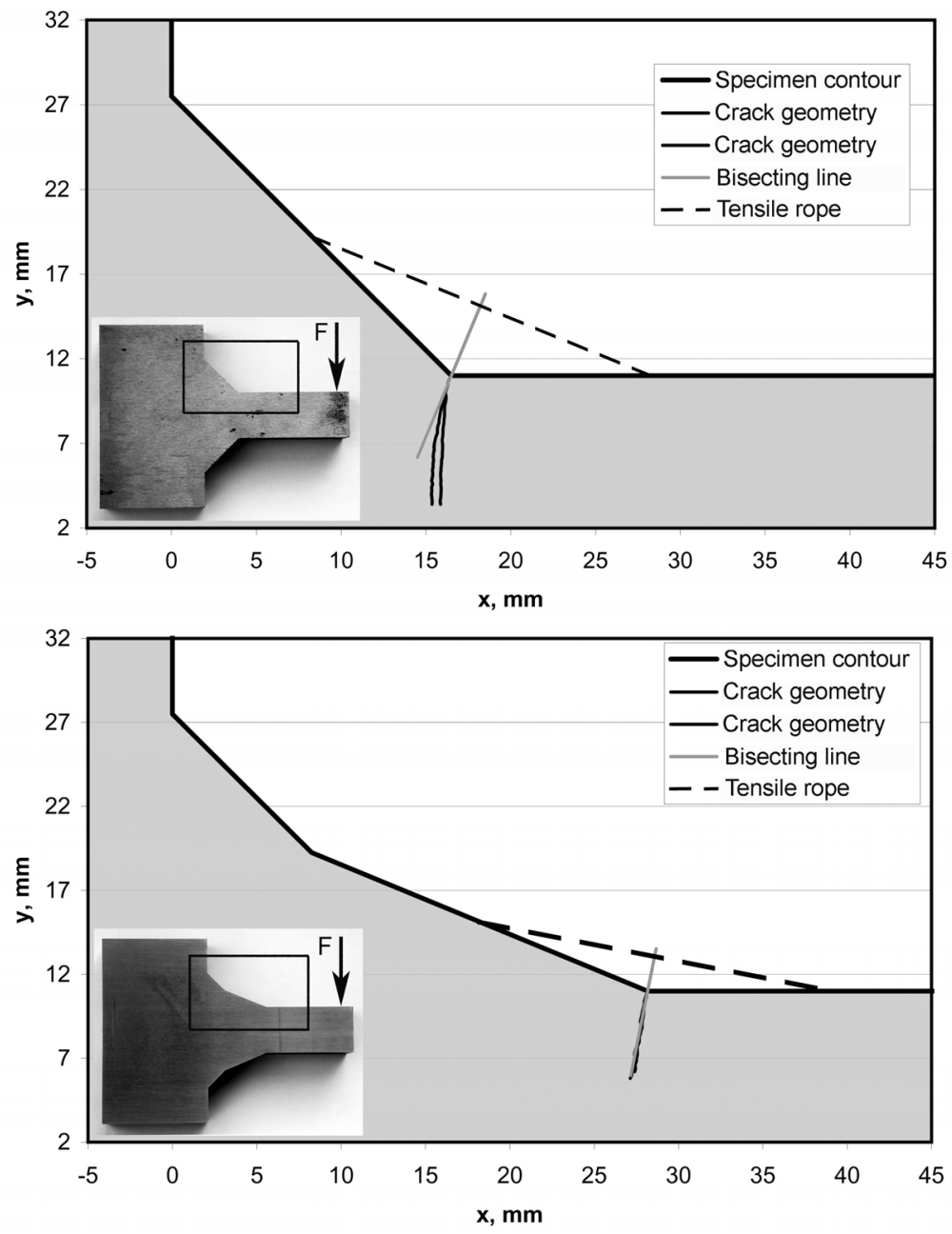

Figure 10: Results of fatigue tests with front- and backside crack path. 
Design and Nature III: Comparing Design in Nature with Science and Engineering 21

\section{References}

[1] C. Mattheck, Engineering structures grow like trees, Materialwissenschaften und Werkstofftechnik, 1990, 143.

[2] C. Mattheck, J. Sörensen, A. Sauer, I. Tesari, Eine graphische Methode zur Kerbformoptimierung, Konstruktionspraxis 2005, 10, 12.

[3] C. Mattheck, K. Bethge, On the Plausibility of the Method of Tensile Triangles (MTT), Materialwissenschaften und Werkstofftechnik, 2005, 36. 